\title{
MINI-GAUGE EPIDURAL NEEDLES AND CATHETERS FOR LABOUR ANALGESIA
}

\begin{abstract}
Pamela Angle MD FRCPC MSc (1), Kamal Hussain MBBS FFARSCI (1); Anwar Morgan MB (1) ; Stephen Halpern MD FRCPC MSc (1); M. Van der Vyver MB (1); Jennifer Yee RN(1), Alexander Kiss PhD (2). Departments of Anesthesia (1) and Research Design \& Biostatistics (2), Sunnybrook \&Women's College Health Sciences Centre, University of Toronto, 76 Grenville Street, Toronto, ON M5S 1B2
\end{abstract}

INTRODUCTION: Needle redesign revolutionized spinal anesthesia by producing large reductions in postdural puncture headache (PDPH). Epidural needles now represent the leading cause of severe PDPH after regional anesthesia. Further success in prevention is most likely to come from use of smaller gauge epidural needles. $20 \mathrm{~g}$ epidural steroid injection has a reported PDPH incidence of $9.8 \%$ (1)compared with $>70 \%$ with larger needles. 20g epidural needles produce a 4-5 fold reduction in CSF leak compared with current needles following puncture(2) and clinical work suggests that blood patch is more successful for smaller gauge punctures (3). This trial examined the feasibility of using mini-gauge Tuohy needles and catheters for continuous epidural labour analgesia.

METHODS: Following REB approval, ASA 1-2 parturients were recruited. Epidural placement involved use of a $19 \mathrm{~g}(19.5 \mathrm{~g}, 90 \mathrm{~mm})$ Tuohy needle (smallest needle permitting $23 \mathrm{~g}$ catheter passage) and a $23 \mathrm{~g}$ single port Portex $40 \mathrm{~cm}$ epidural catheter. Analgesia was initiated using bupivacaine $0.08 \%$ with fentanyl $2 \mathrm{mcg} / \mathrm{ml}(15-20 \mathrm{ml})$ and maintained using PCEA. Breakthrough pain was treated by protocol. There was no formal in-training period for anesthesiologists. Patients were interviewed after epidural initiation, $\leq$ first 24 hours of delivery and between 14-21days post partum. The primary outcome was the combined failure rate (failed insertion or failed block $\leq 30$ minutes of initiation). Secondary outcomes included: pain relief; incidence of puncture, PDPH; mode of delivery, details of epidural placement and anesthesiologist satisfaction.

RESULTS: 27 parturients (13 multiparous) were recruited. Successful block initiation was achieved in 24/27 and maintained throughout labor. Overall pain relief in 24/24 women was good to excellent (19/24 very good to excellent). Three block failures (2 unilateral, 1 absent) occurred (1 per each of three different anesthesiologists) all during initiation phase (early in study recruitment) to give a combined failure rate of $11 \%$ (95\% CI 0.02,0.30). One patient developed PDPH post discharge (reported on day 14 followup) self treated with acetaminophen with complete symptom resolution. Anesthesiologist satisfaction was high.

DISCUSSION: Our work suggests that mini-gauge epidurals may be used to provide high quality analgesia and may reduce morbidity following dural puncture.

\section{REFERENCES:}

1.Anesthesiology $1998 ; 88$ 习 8 8 1

2.Anesthesiology 2003;99: 1376-8 2

3.Anesthesiology 2001; 95: 334-9 